\title{
First Case of Metastasis of a Chordoma in the Abdominal Wall
}

Rocío Santos-Rancaño ${ }^{1 *}$, Carlos Cerdán-Santacruz ${ }^{2}$, Pablo Talavera1 ${ }^{1}$, Tomasz Rogula ${ }^{3}$, Erick Ahnfeldt ${ }^{3}$, Pablo Rodriguez ${ }^{4}$, Karina Antonio ${ }^{5}$, Javier Cerdán-Miguel ${ }^{2}$, Andrés Sánchez-Pernaute ${ }^{5}$ and Antonio J Torres García ${ }^{1}$

${ }^{1}$ Department of General Surgery, San Carlos Clinic Hospital of Madrid, Spain

${ }^{2}$ Department of General Surgery, Santa Elena Clinic, Madrid, Spain

${ }^{3}$ Department of General Surgery, Cleveland Clinic, Cleveland, Ohio, USA

${ }^{4}$ Unit of Abdomen Imaging Diagnosis, Department of Radiology and Imaging, San Carlos Clinic Hospital of Madrid, Spain

${ }^{5}$ Department of Histopathology, San Carlos Clinic Hospital of Madrid, Spain

\begin{abstract}
Chordomas are rare malignant primary bone tumors of ectodermal origin, that arise from notochordal remnants of the developing spine, which most often occur in the sacral area. It is an aggressive, locally invasive neoplasm, which carries a poor prognosis. Chordomas' metastatic incidence ranges from 5 to $40 \%$. It is generally believed that metastases without local recurrence of primary neoplasm are extremely rare. En-bloc excision with wide margins and postoperative radiation therapy remains the only curative treatment of primary chordoma. Its metastases should also be resected surgically.

We present the first case of metastasis of a previously surgically treated primary sacrococcygeal chordoma. Metastatic lesions developed in the abdominal wall of a 42-year-old man without local recurrence and were discovered incidentally. We also describe the surgical management of this case.

This phenomenon has not yet been described. We demonstrate that, incidentally discovered chordoma metastasis in the abdominal wall can occur and present a diagnostic challenge. This case report focuses on the importance of follow up with magnetic resonance imaging or computerized tomography scan after resection of sacral chordoma paying attention to the entire abdomen. After this experience we feel that surgery could be considered a valid option for the treatment of chordoma metastasis in this scenario.
\end{abstract}

Keywords: Chordoma; Metastasis; Abdominal wall; Neoplasm; Sacrum

\section{Background}

A chordoma is a rare, low-grade, primary malignant bone tumor of ectodermal origin derived from cellular remnants of the primitive notochord [1]. Through development, the notochord is lost in areas where it is engulfed by developing vertebral bodies [2]. Notochord remnants within the vertebral bodies may develop into chordomas which can develop anywhere along the spine [2], but have a predilection for the axial skeleton with the most common sites being the sacrococcygeal region (accounting for $50-60 \%$ of all cases), spheno occipital region (mainly the clivus) (25-35\% of cases), and the vertebral column (15\%of cases) $[3,4]$. It accounts for $1-4 \%$ of all primary skeletal tumors and it has the lowest incidence rate at $0.1-0.5$ per 100,000 inhabitants per year [5].

A chordoma is a slow-growing tumor; aggressive, locally invasive, and has a poor prognosis although it has a low and late tendency in metastatizing to distant sites (5-40\%) including the lung, bone, soft tissue, lymph nodes, liver, and skin [6-9].

Its incidence is highest in the sixth decade, but it can also be found in younger population, even infancy (1\%). There is a male-to-female ratio of 2-3:1 [10].

Adequate wide resection still remains the cornerstone of chordoma treatment and its metastases. The gold standard treatment for chordomas of the mobile spine and sacrum is en-bloc excision with wide margins and postoperative external-beam radiation therapy [11]. The achievement of negative surgical margins favorably correlated with the rate of local relapse and survival $[11,12]$.

We present the first case of chordoma metastasis in the abdominal wall. It recurred thirteen months after complete resection of a primary sacral chordoma.

\section{Case Presentation}

A 42-year-old man of Caucasian ethnic background presented with a twelve month history of severe pain in the sacral region and intermittent lower abdominal dull pain radiating to both legs which was not relieved with analgesic medications. The patient had no complaints of urinary incontinence, stool retention, tenesmus, sciatic pain, or paresis of the lower extremities. The patient's medical, surgical, and family history were unremarkable. On his initial physical exam, a rough palpable mass lesion was present over the lower sacral area, approximately $9 \times 10 \mathrm{~cm}$ in size. His rectal examination revealed a dense mass on the posterior wall of the rectum. The rectal tone and perianal examination was normal.

A lumbosacral computed tomography (CT) scan reported an 8.7 $\times 10 \times 8.8 \mathrm{~cm}$ osteolytic, expansible mass involving the sacrum and infiltrating the retrorectal fat tissue partially but not the rectum. It revealed the destruction of sacral vertebras and roots S3, S4 and S5 and

*Corresponding author: Rocio Santos Rancaño, C/o Arzobispo Morcillo, no 12, 70 B, Madrid, Spain, Tel: 0034669781 763; Fax: 0034 913303183; E-mail: rociosantosr@hotmail.com

Received September 08, 2013; Accepted November 11, 2013; Published November 13, 2013

Citation: Santos-Rancaño R, Cerdán-Santacruz C, Talavera P, Rogula T, Ahnfeld $E$, et al. (2013) First Case of Metastasis of a Chordoma in the Abdominal Wall. J Cancer Sci Ther 5: 391-394 doi:10.4172/1948-5956.1000231

Copyright: (C) 2013 Santos-Rancaño R, et al. This is an open-access article distributed under the terms of the Creative Commons Attribution License, which permits unrestricted use, distribution, and reproduction in any medium, provided the original author and source are credited. 
the infiltration of bilateral gluteus maximus but no distant metastasis were seen (Figure 1A).

To complete the study, a magnetic resonance imaging (MRI) was performed and showed the mass previously described destroying the sacrum and growing towards the presacral region (Figure 1B). The mass contained small calcifications (Figure 1A).

The tumor was entirely surgically excised with a partial sacrectomy at S2 level. The coccyx and the bilateral gluteus maximus muscles were also resected with negative surgical margins. The S2 roots were preserved and the pelvic floor reconstruction was performed afterwards with a left rectus flap to close the abdomen with a Prolene $m e s h^{m}$. Macro and micro analysis of the specimen obtained at the time of surgery revealed a dedifferentiated chordoma with negative margins. The patient then underwent postoperative high dose radiation therapy (consisting of a total of $60 \mathrm{~Gy}$ in 27 sessions).

The patient was regularly followed at 3 months intervals with no physical or radiological signs of local or distant recurrence including pelvic MRI and thorax-abdomen-pelvis CT scan. Thirteen months after the surgical resection the patient remained asymptomatic but two solid nodules in contact with the internal side of the left transversalis muscle of the abdominal wall and depending from it $(13 \times 19 \mathrm{~mm}$ and $12 \mathrm{~mm}$ in diameter respectively) without local recurrence of the neoplasm, were seen on a surveillance MRI (Figures $2 \mathrm{~A}-2 \mathrm{C}$ and $2 \mathrm{E}$ ). After this, a CT (Figure 2D) and an ultrasound scans (Figure 2F) were performed and they described the above mentioned nodules. The lungs, liver and other possible sites of metastases were free of disease.

Fine needle puncture aspiration of the nodule was performed with ultrasound guidance, obtaining the diagnosis of chordoma metastases.

Because the patient presented with a local lesion with infiltration of the surrounding muscles and without evidence of other distant
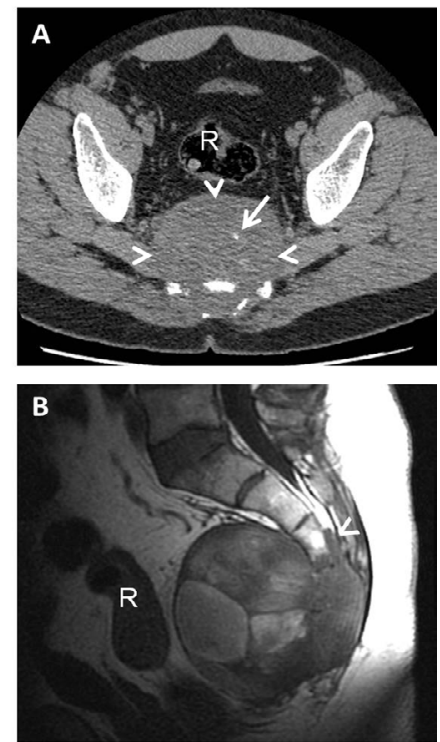

Figure 1: The primary chordoma. (A) Axial non-enhanced CT image. There is a mass (arrowheads) destroying the sacrum and growing towards presacral region. The mass contains small calcifications (arrow). The rectum $(R)$ is located anteriorly. (B) MR Sagital SSFSE T1 weighted image. The MR shows a heterogeneous mass which invades the sacral canal (arrowhead). The mass has high signal intensity contents that probably represent hemorrhagic components. Rectum (R).
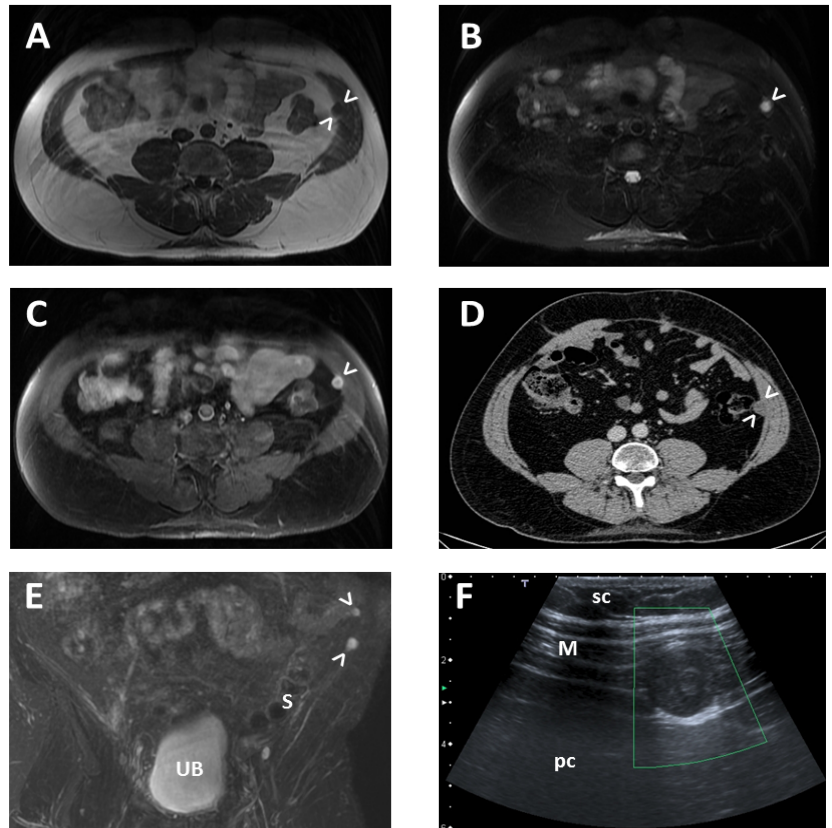

Figure 2: Metastases of chordoma in the abdominal wall. (A) MR Axial SSFSE T1 weighted image. There is a nodule in contact with the internal side of the left transversalis muscle of the abdominal wall (arrowheads). The nodule is hypointense to the muscle and is barely distinguished from the transverse abdominus. (B) MR Axial SSFSE T2 weighted image with fat saturation. The nodule is markedly hyperintense (arrowhead). (C) MR Axial SSFSE $\mathrm{T} 1$ weighted image with fat saturation and intravenous gadolinium contrast injection. The nodule shows a peripheral rim enhancement (arrowhead). (D) Axial enhanced CT image. Nodule in contact with the internal side of the left transversalis muscle (arrowheads). (E) MR Coronal SSFSE T2 weighted image with fat saturation. The two abdominal wall metastases are pointed with arrowheads. Urinary bladder (UB) and sigmoid colon (S). (F) Doppler ultrasound image of one of the abdominal wall nodules, obtained with a linear transducer. There is no evidence of significant flow inside the nodule. Abdominal muscle wall (M), subcutaneous tissue (sc) and peritoneal cavity (pc).

dissemination, wide local resection was chosen. The metastatic nodules were excised entirely laparoscopically. Both nodules were seen as protruding over the peritoneum without invasion, developed in the transversalis abdominal muscle. Laboratory studies, including a hemogram and biochemistry examinations, were unremarkable.

Conventional photon beam radiation therapy was delivered in high doses (60 Gy in 25 sessions) in the metastasic field to obtain a curative effect.

Metastasis of chordoma in the abdominal wall was histopathologically confirmed in the analyses of the surgical specimen (Figure 3). No local or distant recurrence has been observed one year after its surgical resection. The patient is regularly followed-up in 3 months intervals consisting in pelvic MRI and thorax-abdomen-pelvis CT.

\section{Discussion}

The first case of a chordoma characterized microscopically was in 1857 by Virchow [13]. Although histologically considered to be a lowgrade neoplasm, chordomas are highly recurrent and are very locally aggressive, making their clinical progression very similar to that of malignant tumors. Although metastases are rare [2-4,14], they still have poor long term prognosis. 

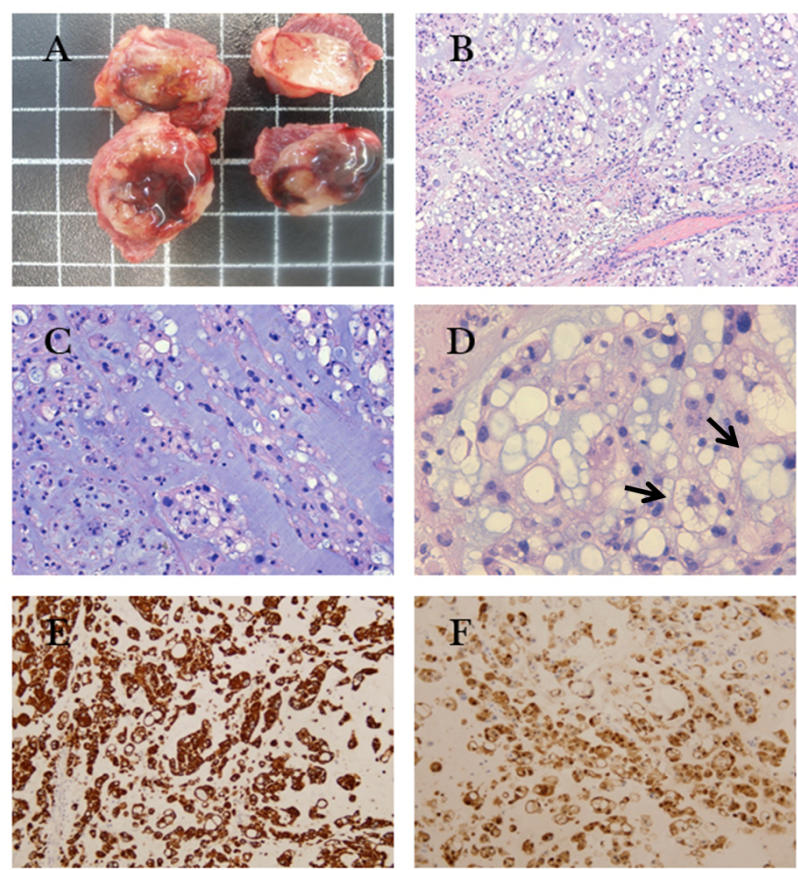

Figure 3: Histopathological analyses of the surgical specimen. Metastatases of chordoma in the abdominal wall (A) Gross image of chordoma showing a lobulated and well-defined tumor. (B) Hematoxylin and eosin-stained sections of tumor with a lobular growth pattern (X20). (C) Abundant basophilic to metachromatic mucinous or myxoid stroma comprises the majority of the tumor volume. The cells of chordoma are round to ovoid with pale eosinophilic cytoplasm growing in strands, cords, and clusters (X200) (D) Physaliphorous cells are large cells with multiple vacuoles in the cytoplasm $(X 400)$. (E) In this case the neoplastic cells are immunoreactive strongly with CKAE1/AE3, and (F) protein S-100 in a weak and patched (X200).

In a recent study, a 5 -year survival rate of $67 \%$ and 10 -year survival rate of $40 \%$ were reported [15]. Patient survival seems to be less affected by distant metastasis than by local progression of the disease [16]. Local recurrence has emerged as the most important predictor of mortality in patients with chordoma, and the extent of initial resection has become the most compelling factor concerning the opportunity for a cure [17].

According to the literature, almost $17-35 \%$ of the patients with chordoma present signs of local recurrence within various period of time after surgery. Distant metastases have a poor prognosis; its incidence varies from $3-48 \%$ of cases [17]. Kishimoto et al. reviewed 198 patients with chordoma and reported a metastatic incidence of $24.7 \%$ [9]. The incidence of metastases in sacroccocygeal chordomas is estimated at $17.5 \%$ of cases [4]. Metastases mostly occur in patients with sacrococcygeal or vertebral tumors and are very unlikely in cases without local recurrence [11]. The most common places in descending order of occurrence include lung (48\%), lymph nodes, liver, bone, skin, muscles, peritoneum, kidney, spleen and heart [15]. This is why MRI and CT surveillance of all such patients are imperative after the primary surgery, particularly in cases of proven aggressive biological behavior of the primary neoplasm. Very few authors describe metastases in the skin $[7,8]$, lymphatics $[6]$ or in the heart $[18,19]$ but to our best knowledge, this is the first case in the literature that describes metastases of a chordoma in the abdominal wall.

In the imaging tests of the primary chordoma, bone destruction, calcifications, osteosclerosis in the nearby bone and the presence of a soft tissue mass with osseous expansion are findings that can be seen $[9,20]$. CT and MRI studies (specially T2 weighted images) are indicated to evaluate the extent of the tumor, to identify the tissues that the tumor has infiltrated, to rule out metastases and for preoperative planning purposes [20,21], although definitive diagnostic certainty is based on histological examination. Diffusion weighted images (DWI) has proved to be useful for detecting metastases [9], unfortunately we did not acquired DWI in our patient. For the follow up of patients with chordoma also CT and MR imaging have shown to be useful.

Fine-needle aspiration biopsy has been suggested to be the most oncologically approach to establish a diagnosis before resection, with care to avoid tumor seeding [4].

Clinical signs and symptoms may vary depending on the location, size of tumor, extent of tissue invasion and presence of metastases.

The literature provides different suggestions and experiences about surgical approaches, but no method has become a standard due to the heterogeneity of these tumors. There is some consensus that radiation therapy increase disease-free intervals [22]. Current standard treatment options include surgery and radiation therapy, with gross en bloc excision offering the best chance for long-term survival [11]. This approach is also the best for the recurrences of metastases [3,4]. Radiotherapy is often used in high doses, as chordomas are sometimes resistant to this treatment.

Sensitivity to chemotherapy is very low and generally reported in the small subgroup of patients with high-grade dedifferentiated chordomas using agents active in high-grade sarcomas. Targeted molecular therapeutic drugs now under development may be used to supplement surgery [23]. Imaging-guided percutaneous radiofrequency ablation was recently described as a possible alternative to surgery for locally recurrent disease or for palliation [23]. Our patient underwent a total resection with partial sacrectomy followed by adjuvant radiation therapy. For the metastases we performed a wide local surgical excision.

In conclusion, the importance and relevance of this extraordinary case is a new localization of distant chordoma metastasis, incidentally discovered in the abdominal wall, in the transversalis muscle, a location that has never been described before.

This case report focuses on the importance of a rigorous follow up with MRI and/or CT after resection of a sacral chordoma paying attention to the entire abdomen. Correct diagnosis requires vigilance, radiological, clinical and pathological correlation and careful attention and suspicion of this possible metastatic location. After this experience we believe that surgery could be considered a valid option for the treatment of chordoma metastasis in this location.

\section{Consent}

Written informed consent was obtained from the patient for publication of this Case report and any accompanying images. A copy of the written consent is available for review by the Editor of this journal.

\section{Competing Interests}

The authors declared that they have no competing interests

\section{Acknowledgements}

RS and CC drafted the manuscript and were members of the clinical team. PT, RS carried out the clinical management of the patient. PR provided expert advice, analysis and images. KA carried out pathologic assessments. JC and AJT participated in the design and coordination of the report. EA, TR supervised 
Citation: Santos-Rancaño R, Cerdán-Santacruz C, Talavera P, Rogula T, Ahnfeldt E, et al. (2013) First Case of Metastasis of a Chordoma in the Abdominal Wall. J Cancer Sci Ther 5: 391-394. doi:10.4172/1948-5956.1000231

and helped to draft the manuscript. All authors provided substantive intellectual contributions, read and approved the final manuscript.

\section{References}

1. Rossleigh MA, Smith J, Yeh SD (1986) Scintigraphic features of primary sacral tumors. J Nucl Med 27: 627-630.

2. Vergara G, Belinchon B, Valcarcel F, Veiras M, Zapata I, et al. (2008) Metastatic disease from chordoma. Clin Transl Oncol 10: 517-521.

3. Ferraresi V, Nuzzo C, Zoccali C, Marandino F, Vidiri A, et al. (2010) Chordoma: clinical characteristics, management and prognosis of a case series of 25 patients. BMC Cancer 10: 22.

4. Walcott BP, Nahed BV, Mohyeldin A, Coumans JV, Kahle KT, et al. (2012) Chordoma: current concepts, management, and future directions. Lancet Oncol 13: e69-76.

5. Jemal A, Siegel R, Ward E, Murray T, Xu J, et al. (2007) Cancer statistics. CA Cancer J Clin 57: 43-66.

6. Sopta J, Tulic G, Mijucic V, Mamontov P, Mandic N (2009) Solitary lymph node metastasis without local recurrence of primary chordoma. Eur Spine J 18: 191 195

7. Collins GR, Essary L, Strauss J, Hino P, Cockerell CJ (2012) Incidentally discovered distant cutaneous metastasis of sacral chordoma: a case with variation in S100 protein expression (compared to the primary tumor) and review of the literature. J Cutan Pathol 39: 637-643.

8. Rubin Al, Bagel J, Niedt G (2005) Chordoma cutis. J Am Acad Dermatol 52 S105-108.

9. Kishimoto R, Omatsu T, Hasegawa A, Imai R, Kandatsu S, et al. (2012) Imaging characteristics of metastatic chordoma. Jpn J Radiol 30: 509-516.

10. Shinmura Y, Miura K, Yajima S, Tsutsui Y (2003) Sacrococcygeal chordoma in infancy showing an aggressive clinical course: an autopsy case report. Pathol Int 53: 473-477.

11. McPherson CM, Suki D, McCutcheon IE, Gokaslan ZL, Rhines LD, et al. (2006) Metastatic disease from spinal chordoma: a 10-year experience. J Neurosurg Spine 5: 277-280.
12. Boriani S, Bandiera S, Biagini R, Bacchini P, Boriani L, et al. (2006) Chordoma of the mobile spine: fifty years of experience. Spine (Phila Pa 1976) 31: 493503.

13. Virchow RL (1857) Untersuchungen ueber die Entwicklung des Schaedelgrundes, Berlin, G Rimer.

14. Schwab JH, Boland PJ, Agaram NP, Socci ND, Guo T, et al. (2009) Chordoma and chondrosarcoma gene profile: implications for immunotherapy. Cancer Immunol Immunother 58: 339-349.

15. Hsieh PC, Xu R, Sciubba DM, McGirt MJ, Nelson C, et al. (2009) Long-term clinical outcomes following en bloc resections for sacral chordomas and chondrosarcomas: a series of twenty consecutive patients. Spine (Phila Pa 1976) 34: 2233-2239.

16. Fletcher CD (2006) The evolving classification of soft tissue tumours: an update based on the new WHO classification. Histopathology 48: 3-12.

17. York JE, Kaczaraj A, Abi-Said D, Fuller GN, Skibber JM, et al. (1999) Sacra chordoma: 40-year experience at a major cancer center. Neurosurgery 44: 74 79.

18. Ruiz HA, Goldberg LH, Humphreys TR, Blacklock JB (2000) Cutaneous metastasis of chordoma. Dermatol Surg 26: 259-262.

19. Prompona M, Linn J, Burdorf L, Assmann G, Reichart B, et al. (2009) Pulmonary vein metastasis of a sacral chordoma extending into the left atrial cavity. J Cardiovasc Med (Hagerstown) 10: 557-559.

20. Gerber S, Ollivier L, Leclère J, Vanel D, Missenard G, et al. (2008) Imaging of sacral tumours. Skeletal Radiol 37: 277-289.

21. Plathow C, Weber MA, Debus J, Kauczor HU (2005) [Imaging of sacral chordoma: comparison between MRI and CT]. Radiologe 45: 63-68.

22. Forsyth PA, Cascino TL, Shaw EG, Scheithauer BW, O'Fallon JR, et al. (1993) Intracranial chordomas: a clinicopathological and prognostic study of 51 cases. J Neurosurg 78: 741-747.

23. Muro K, Das S, Raizer JJ (2007) Chordomas of the craniospinal axis: multimodality surgical, radiation and medical management strategies. Expert Rev Neurother 7: 1295-1312. 